\title{
El uso de bioensayos crónicos en Daphnia magna para la evaluación ambiental de un arroyo urbano en Tierra del Fuego (Argentina)
}

\author{
Antonela Albizzi' Soledad Diodato ${ }^{12,2}$ \& Gabriela González Garraza ${ }^{1,2}$ \\ ${ }^{1}$ Centro Austral de Investigaciones Científicas (CADIC), CONICET. Ushuaia, Tierra del Fuego, Argentina. ${ }^{2}$ Instituto de \\ Ciencias Polares, Ambiente y Recursos Naturales (ICPA), Universidad Nacional de Tierra del Fuego (UNTDF). Ushuaia,
} Tierra del Fuego, Argentina.

\begin{abstract}
Resumen. El desarrollo de la urbanización impacta negativamente sobre los ambientes acuáticos, ya que los convierte en receptores finales de una variedad de sustancias antrópicas, muchas de ellas causantes de eutrofización. En Ushuaia (Tierra del Fuego), el Arroyo Buena Esperanza (ABE) ha sido sometido a estos impactos, habiéndose detectado que la calidad del agua disminuye a medida que el arroyo atraviesa el ejido urbano y desemboca en la Bahía Encerrada (BE). Sin embargo, se desconoce si presenta toxicidad sobre la biota. Evaluamos la calidad del agua del ABE a lo largo de su recorrido por el ejido urbano y en su desembocadura en la BE mediante el efecto sobre la supervivencia (48 horas) y el crecimiento (21 días) de Daphnia magna. Realizamos bioensayos agudos y crónicos con neonatos de D. magna expuestos a aguas de cuatro sitios del ABE: uno sin impacto urbano, uno con impacto intermedio y dos con impacto alto. La calidad del agua disminuyó a medida que el arroyo atraviesa el ejido urbano, con valores elevados de amonio, fosfatos, materia orgánica particulada, sólidos totales en suspensión y bacterias coliformes (por el aporte de efluentes cloacales sin tratar). La supervivencia de D. magna a las 48 horas fue cercana al $100 \%$, lo cual indica la ausencia de toxicidad aguda del agua. La biomasa individual varió según el sitio y el tiempo ensayado; aumentó gradualmente, pero no de forma pareja entre los sitios. Los organismos expuestos a los sitios más impactados presentaron mayor biomasa y tasa de crecimiento en comparación con los sitios con menor impacto. Esto se vincula a la peor calidad del agua de los sitios impactados. El crecimiento de D. magna resultó ser una herramienta de diagnóstico ambiental aplicable para detectar efectos subletales de las aguas de arroyos fueguinos, y un complemento adecuado del monitoreo ambiental.
\end{abstract}

[Palabras clave: ecotoxicidad, impacto urbano, microcrustáceos, biomasa individual, $\mathrm{CL}_{50}$ ]

Aвstract. The use of chronic bioassays in Daphnia magna for the environmental evaluation of an urban stream in Tierra del Fuego (Argentina). The development of urbanization has a negative impact on aquatic environments, making them the final receptors for a variety of anthropic substances, many of which lead to eutrophication. In Ushuaia (Tierra del Fuego), the Arroyo Buena Esperanza (ABE) has been subjected to these impacts. Its water quality decreases as the stream crosses the urban zone and discharges into Bahía Encerrada (BE). However, it is unknown whether its waters are toxic to the biota. We evaluated the water quality of the $\mathrm{ABE}$ all along its path through the urban zone and at its mouth in the BE through the effect on survival (48 hours) and growth (21 days) of Daphnia magna. Acute and chronic bioassays were conducted with D. magna neonates exposed to waters from four sites of the ABE: one with no urban impact, one with intermediate impact, and two with high impact. Water quality decreased as the stream crosses the urban zone, finding high values of ammonia, phosphates, particulate organic matter, total suspended solids and coliform bacteria; caused by the contribution of untreated sewage effluents. The survival of D. magna at 48 hours was close to $100 \%$, which indicates the absence of acute toxicity of the water. The individual biomass varied according to the site and the time tested, increasing gradually but not evenly between sites. The organisms exposed to the most impacted sites presented higher biomass and growth rate in comparison to the sites with the least impact. This is linked to the poorer water quality of the impacted sites. The growth of $D$. magna was an environmental diagnostic tool with high applicability for the detection of sublethal effects of the waters of Fuegian streams, which adequately complements the environmental monitoring.

[Keywords: ecotoxicity, urban impact, microcrustaceans, individual biomass, $\mathrm{LC}_{50}$ ] 


\section{INTRODUCCIÓN}

El desarrollo y crecimiento de las áreas urbanas pueden generar impactos en forma directa sobre los cuerpos fluviales aledaños (Angerville et al. 2013; Becouze-Lareure et al. 2016; van Leeuwen et al. 2019) a través del vertido de aguas residuales domiciliarias y provenientes del sistema de alcantarillado. En zonas donde los cursos fluviales atraviesan bosques es común observar procesos de eutrofización natural producto de los aportes alóctonos de materia orgánica (Le Moal et al. 2019). No obstante, si el curso de agua atraviesa, además, un ejido urbano, los aportes antrópicos pueden causar efectos adversos sobre la calidad fisicoquímica del agua, los sedimentos y la biota (Chalmers et al. 2007; Göbel et al. 2007; Angerville et al. 2013). Dichos efectos se relacionan con el aumento de la turbidez por la presencia de material en suspensión, la desoxigenación del ambiente causada por el ingreso de materia orgánica degradable y los efectos tóxicos sobre los organismos acuáticos. Es así que los cursos de agua dulce que nacen en la zona boscosa sobre la montaña y atraviesan un casco urbano se convierten en las vías directas de transporte de sustancias originadas por desechos de la urbanización y las naturales (Diodato et al. 2018; Le Moal et al. 2019); finalmente, esas sustancias impactan en el sistema costeromarino.

La ciudad de Ushuaia $\left(54^{\circ} 48^{\prime} 26^{\prime \prime} \mathrm{S}\right.$ $68^{\circ} 18^{\prime} 16^{\prime \prime} \mathrm{O}$ ), en la provincia argentina de Tierra del Fuego, emplazada en la costa norte del Canal Beagle, experimentó un extraordinario desarrollo demográfico en las últimas décadas (de 7000 habitantes en 1970 a 60000 en 2010) (Censo Nacional 2010). Las necesidades sanitarias no fueron atendidas con una planificación eficiente de los recursos e infraestructura, lo cual tuvo como consecuencia el vertido de efluentes domiciliarios crudos sobre los arroyos urbanos y la costa durante décadas. Sin embargo, en los últimos tres años se llevaron adelante obras de infraestructura tendientes a solucionar esta problemática, con lo que disminuyó en gran medida el aporte de efluentes a los cursos de agua. Aun así, los cuerpos de agua urbanos todavía reciben una cierta cantidad de aguas residuales que continúan impactando en el sistema.

Existen numerosos trabajos que dan cuenta del impacto de la urbanización sobre los cursos de agua y la zona costera de la ciudad de Ushuaia (Torres et al. 2009; Amin et al. 2011; Diodato et al. 2012; Diodato 2013; Dirección General de Recursos Hídricos 2015; Zagarola et al. 2017; Diodato et al. 2018, 2020; Granitto et al. 2021). La calidad del agua es buena en las zonas altas de las cuencas del Arroyo Grande, Arroyo Buena Esperanza, Río Pipo y Río Olivia, aguas arriba de la urbanización, pero disminuye significativamente por el aporte urbano que recibe en su trayecto hacia las desembocaduras en la zona costera. Todos estos estudios demuestran que los sitios más impactados, con evidencia de eutrofización, registran alta concentración de bacterias coliformes fecales, lo cual indica una clara contaminación por materia fecal.

Los bioensayos de toxicidad son una herramienta de diagnóstico que permiten determinar el efecto de agentes fisicoquímicos y contaminantes sobre la mortalidad, el crecimiento y la reproducción de organismos de prueba (Castillo Morales 2004; Ferrari 2006; Scholes et al. 2007; Marcogliese et al. 2015). Con frecuencia se recurre a especies planctónicas como organismos de prueba, y particularmente a los cladóceros, dado que son un componente fundamental de la comunidad zooplanctónica que funciona como el enlace principal entre los productores primarios y los consumidores de niveles tróficos superiores (Martínez-Jerónimo et al. 2008). Dentro de los cladóceros, Daphnia magna (Strauss, 1820) es una de las especies más utilizadas (USEPA 2002; Damásio et al. 2008; Giraudo et al. 2019).

Implementar esta perspectiva ecotoxicológica permite complementar de forma rápida y sencilla los estudios de monitoreo de parámetros fisicoquímicos indicadores de calidad de agua con las respuestas de los organismos, evaluandoposiblesconsecuencias, por ejemplo, sobre la supervivencia y el crecimiento (Ferrari 2006; Gopi et al. 2012; Giraudo et al. 2019). En este contexto, el objetivo de este trabajo fue evaluar la calidad del agua del Arroyo Buena Esperanza (ABE) a lo largo de su recorrido por el ejido urbano de la ciudad de Ushuaia, mediante su efecto en la supervivencia y en el crecimiento de Daphnia magna. Para llevar a cabo dicho objetivo se estudió simultáneamente la calidad del agua desde el punto de vista físico, químico y microbiológico en cuatro sitios del ABE, y su toxicidad aguda (a 48 horas) y crónica (a 21 días) sobre los microcrustáceos. 


\section{Materiales y Métodos}

\section{Área de estudio}

El área de estudio abarca la cuenca fluvial del Arroyo Buena Esperanza (ABE), un curso de agua que nace en el valle Martial, Cordillera Fueguina, a 1340 m s. n. m., atraviesa el ejido urbano de la ciudad y desemboca en la Bahía Encerrada (BE), en la zona costera del Canal Beagle. Sus aguas son hiposalinas, ligeramente bicarbonatadas, de gran transparencia y con un contenido alto de hierro; la turbidez aumenta durante las crecidas por fusión nival o precipitaciones intensas debido al arrastre de sedimentos (Iturraspe 2007). El caudal medio de este arroyo varía entre 0.3 y $3 \mathrm{~m}^{3} / \mathrm{s}$ dependiendo de las lluvias y eventos de deshielo (Torres et al. 2009). Después de un recorrido de $\sim 7 \mathrm{~km}$ en el cual atraviesa el ejido urbano de la ciudad (Urciuolo and Iturraspe 2005), en sus tramos medio y final es donde recibe la mayoría de las descargas pluvio-cloacales, agua de escurrimiento de turbales y otros pequeños chorrillos. La $\mathrm{BE}$ es una reserva natural urbana lindante con la Bahía Ushuaia, que recibe aportes de agua con distintos niveles de contaminación a través de varios conductos pluviales. El aporte mayor lo recibe mediante el caudal del $\mathrm{ABE}$, mientras que los otros pequeños aportes son descargados a través de caños de menor calibre, con un caudal promedio de $0.01 \mathrm{~m}^{3} / \mathrm{s}$ (Torres et al. 2009).

\section{Diseño de muestreo}

Para colectar las muestras de agua a utilizar en los bioensayos de toxicidad se seleccionaron cuatro sitios: tres a lo largo del ABE y uno en la BE (Figura 1). El primer punto se ubica en el tramo alto (sitio cercano a la cabecera del arroyo, rodeado de un bosque mixto de Nothofagus, sin impacto urbano apreciable [ABEa]); el segundo punto, en el tramo medio (dentro del ejido urbano, con impacto intermedio [ABEm]); el tercer punto, en la desembocadura del arroyo sobre la BE (con alto impacto urbano [ABEd]), y el cuarto punto se ubica dentro de la BE (luego de la desembocadura del ABE en una zona de acumulación de sustancias descargadas por el arroyo [BE]). Los sitios fueron muestreados en dos ocasiones durante la primavera austral de 2019. En cada ocasión, los cuatro sitios de muestreo se muestrearon durante el mismo día, con una diferencia máxima de tres horas de recolección entre el primero y el cuarto sitio.

En cada sitio se registró in situ temperatura, $\mathrm{pH}$, oxígeno disuelto y conductividad

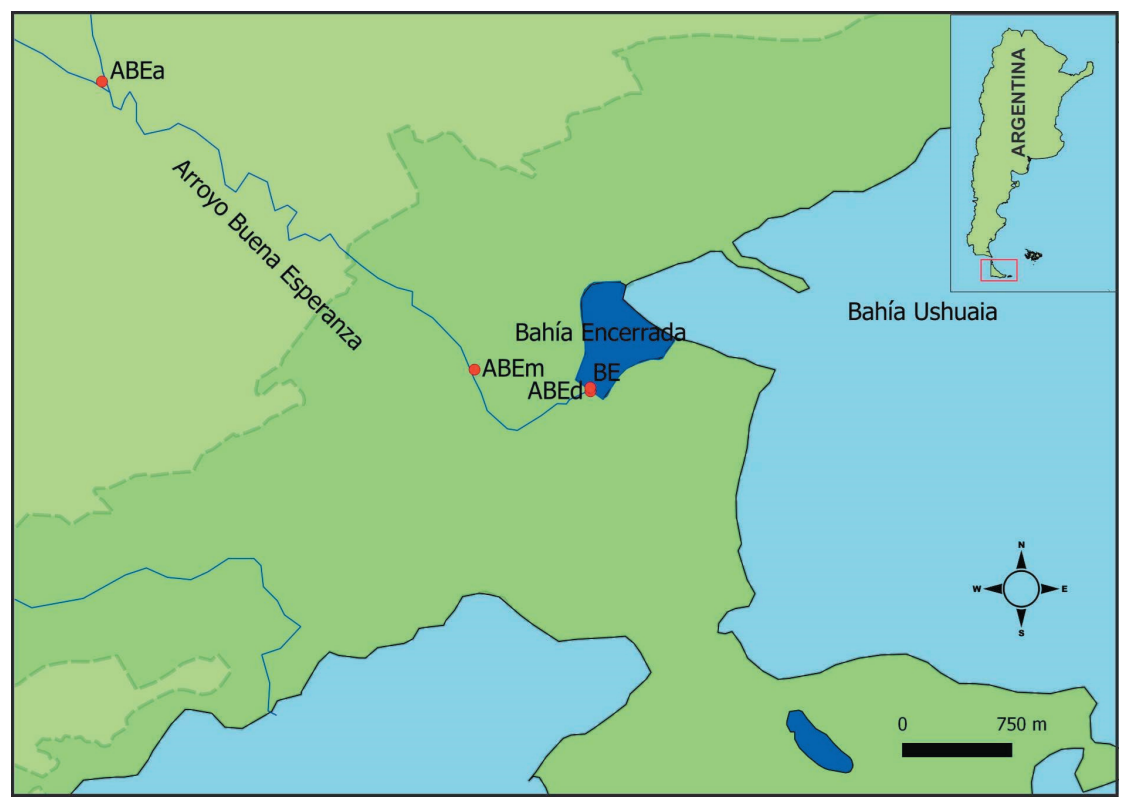

Figura 1. Área de estudio (Ushuaia, Tierra del Fuego, Argentina) y sitios de muestreo sobre el Arroyo Buena Esperanza $(\mathrm{ABE})$ : tramo alto $(\mathrm{ABEa})$, tramo medio $(\mathrm{ABEm})$ y desembocadura (ABEd). El cuarto punto se localiza sobre la desembocadura del ABE en la Bahía Encerrada (BE).

Figure 1. Study area (Ushuaia, Tierra del Fuego, Argentina) and sampling sites in the Arroyo Buena Esperanza (ABE): upper section (ABEa), middle section (ABEm) and mouth (ABEd). The fourth point is located on the mouth of the $\mathrm{ABE}$ in Bahía Encerrada (BE). 
utilizando una sonda multiparamétrica Horiba U-51. Las muestras de agua para determinar los parámetros químicos y para utilizar en los bioensayos de toxicidad fueron sub-superficiales y se colectaron en envases plásticos. Además, se tomaron muestras para la determinación de bacterias coliformes totales (CT) y fecales (CF) en envases estériles. Una vez en el laboratorio, las muestras fueron almacenadas en oscuridad a $4{ }^{\circ} \mathrm{C}$ hasta su inmediato procesamiento (APHA-AWWAWEF 2017).

Caracterización ambiental. Los parámetros químicos utilizados como indicadores de la calidad ambiental fueron nutrientes inorgánicos disueltos, materia orgánica particulada (MOP) y sólidos totales en suspensión (STS). Las muestras para determinar nutrientes inorgánicos disueltos (nitratos, nitritos, amonio, fosfatos y silicatos) y MOP se filtraron a través de filtros de fibra de vidrio de $1.2 \mu \mathrm{m}$ de poro (Whatman ${ }^{\circledR} \mathrm{GF} /$ C) previamente calcinados a $450-500{ }^{\circ} \mathrm{C}$, de acuerdo con Strickland y Parsons (1972). Las muestras de agua filtrada se preservaron a -20 ${ }^{\circ} \mathrm{C}$ hasta el momento de su análisis (APHAAWWA-WEF 2017). Las concentraciones de nitratos $\left(\mathrm{N}-\mathrm{NO}_{3}^{-}\right)$, nitritos $\left(\mathrm{N}-\mathrm{NO}_{2}{ }^{-}\right)$, fosfatos $\left(\mathrm{P}_{-} \mathrm{PO}_{4}{ }^{3-}\right)$ y silicatos $\left(\mathrm{Si}-\mathrm{SiO}_{2}\right)$ se determinaron por duplicado utilizando kits de reactivos Hach ${ }^{\circledR}$, midiendo con un espectrofotómetro Perkin Elmer Lambda 25 y comparando las absorbancias obtenidas con las respectivas curvas de calibración con solución estándar para cada nutriente. La concentración de amonio $\left(\mathrm{N}-\mathrm{NH}_{4}^{+}\right)$se determinó por duplicado siguiendo la técnica del indofenol (Strickland and Parsons 1972) mediante la utilización del kit de reactivos de Wiener®. La concentración de MOP se determinó por duplicado mediante espectrofotometría siguiendo el método de oxidación húmeda de Strickland y Parsons (1972). Para determinar los STS se filtró por duplicado un volumen conocido de agua utilizando membranas de acetato de celulosa Millipore ${ }^{\circledR}$ de $0.45 \mu \mathrm{m}$ de poro, y se aplicó el método gravimétrico para determinar la concentración final (Strickland and Parsons 1972). Para determinar bacterias CT y bacterias CF (Escherichia coli), el cultivo se inició inmediatamente después de recolectar la muestra. La detección y cuantificación se realizó mediante la técnica de fermentación por tubos múltiples ó número más probable (NMP) utilizando la prueba de reactivos Colilert (Idexx) aprobada por USEPA (APHAAWWA-WEF 2017).
Para evaluar la existencia de diferencias significativas de las variables ambientales entre sitios de estudio se realizaron análisis de varianza (ANOVA) de un factor para cada variable, con contrastes a posteriori (Fisher). Previamente, se analizó la normalidad (Shapiro) y la homocedasticidad. Para las variables que no siguieron una distribución normal se realizó la prueba no paramétrica de Kruskal-Wallis. Todos los análisis se realizaron considerando un $P<0.05$ como criterio de significancia, por medio del software Infostat.

Cultivo y mantenimiento de Daphnia magna. Adultos de D. magna fueron obtenidos a partir de un cultivo estable y en óptimas condiciones provisto por la Universidad Nacional de La Plata (Argentina). Para obtener neonatos, los cultivos se mantuvieron en una cámara climatizada a temperatura $\left(15 \pm 2{ }^{\circ} \mathrm{C}\right)$ y fotoperíodo (16:8 luz:oscuridad) constantes. El medio de cultivo utilizado fue agua dura reconstituida de acuerdo con Díaz Báez et al. (2004). Los recipientes de vidrio utilizados para el cultivo fueron limpiados de forma periódica para evitar la acumulación de algas, y se retiraron mudas y organismos muertos. El medio de cultivo fue renovado parcialmente cada 15 días, o totalmente de ser necesario.

Para alimentar a los organismos se aislaron microalgas de un cuerpo de agua dulce local y se las cultivó en medio de Bristol modificado de acuerdo con Chantanachat y Bold (1962) a temperatura ambiente de $22-24{ }^{\circ} \mathrm{C}$, fotoperíodo 12:12 y con aireación constante. Estas microalgas del género Scenedesmus, fueron suministradas a una densidad de $1.15 \times 10^{5}$ individuos/mL junto con levadura seca comercial disuelta para favorecer la nutrición del cultivo (Ten Berge 1978; CastroMejía et al. 2016), tres veces por semana.

Bioensayos de toxicidad con D. magna. Los bioensayos de toxicidad se llevaron a cabo de acuerdo con el protocolo estandarizado de USEPA (2002). Se realizaron tres tipos de bioensayos: de toxicidad aguda con un tóxico de referencia, de toxicidad aguda con muestras de agua del $A B E$ y la BE, y de toxicidad crónica con agua del ABE y la BE. El día anterior al inicio de cada bioensayo se extrajeron los juveniles de D. magna de los recipientes de cultivo para disponer al día siguiente exclusivamente de neonatos menores a 24 horas de edad.

Para evaluar si los individuos del cultivo se mantenían en condiciones fisiológicas óptimas 
para el desarrollo de pruebas de toxicidad se realizó un análisis de la sensibilidad del cultivo empleando dicromato de potasio $\left(\mathrm{K}_{2} \mathrm{Cr}_{2} \mathrm{O}_{7}\right)$ como tóxico de referencia (USEPA 2002; Díaz Báez et al. 2004). Se realizaron cinco ensayos con $\mathrm{K}_{2} \mathrm{Cr}_{2} \mathrm{O}_{7}$, empleando seis tratamientos por triplicado: cinco concentraciones del tóxico y un control (medio de cultivo libre de $\mathrm{Cr}^{6+}$ detectable). Partiendo de una solución madre de $\mathrm{K}_{2} \mathrm{Cr}_{2} \mathrm{O}_{7}$ de 100 $\mathrm{mg} \mathrm{Cr}{ }^{6+} / \mathrm{L}$, las concentraciones reales de $\mathrm{Cr}^{6+}$ utilizadas fueron $0.027 \mathrm{mg} / \mathrm{L}, 0.122 \mathrm{mg} / \mathrm{L}, 0.305$ $\mathrm{mg} / \mathrm{L}, 0.645 \mathrm{mg} / \mathrm{L}$ y $0.724 \mathrm{mg} / \mathrm{L}$, las cuales se determinaron mediante un kit de reactivos Hanna (Cromo (VI), Rango Alto 0 a 1000 gg/L (como $\mathrm{Cr}(\mathrm{VI})$ ), resolución: $1 \mu \mathrm{g} / \mathrm{L}$ ) utilizando un fotómetro multiparámetro Hanna HI-83399. Cada repetición contó con diez neonatos de $D$. magna seleccionados al azar en un volumen de $30 \mathrm{~mL}$ de solución en vasos de precipitado de vidrio. Los individuos fueron expuestos al tóxico por 48 horas, a la misma temperatura $\mathrm{y}$ fotoperíodo que en cultivo y en ausencia de alimento. A las 24 y 48 horas se registró la supervivencia dando por muertos a aquellos individuos inmóviles que no reaccionaban a estímulos (Castillo Morales 2004; Alves and Silvano 2006).

Para evaluar la calidad del agua del ABE y la BE se realizaron cinco ensayos de toxicidad aguda exponiendo durante 48 horas neonatos a muestras de los diferentes sitios seleccionados en el ABE (ABEa, ABEm y ABEd) y la BE, y un control con medio de cultivo. Según la cantidad de neonatos disponibles, cada tratamiento constó de tres o cuatro repeticiones. Cada bioensayo se realizó utilizando 10 neonatos en $30 \mathrm{~mL}$ de agua por repetición, bajo las mismas condiciones controladas de cultivo (i.e., igual temperatura y fotoperíodo) (Castillo Morales 2004). Se estimó la concentración letal $50\left(\mathrm{CL}_{50}\right)$ a 24 y 48 horas de acuerdo con el método probit, utilizando el programa PriProbit (PReference Index Probit) ver. 1.63 (Kyoto University), aprobado por la Environmental Protection Agency (EEUU). Además de la $\mathrm{CL}_{50^{\prime}}$ se calcularon los límites inferior y superior de confianza al $95 \%$ y el coeficiente de correlación para cada ensayo.

Se realizaron dos ensayos de toxicidad crónica utilizando D. magna, cada uno de 21 días de duración para evaluar el crecimiento a través del aumento de la biomasa individual (OECD 2008; Giraudo et al. 2015, 2019). En cada ensayo se utilizaron neonatos expuestos a cinco tratamientos: las muestras de agua de los cuatro sitios de estudio (ABEa, ABEm,
ABEd y BE) más el control de medio de cultivo, cada uno con cuatro repeticiones. En cada repetición se colocaron diez individuos en vasos de precipitado de vidrio con $30 \mathrm{~mL}$ de la muestra correspondiente. Tres veces por semana se renovó totalmente el agua para cada repetición y tratamiento. Los individuos fueron alimentados diariamente con $0.5 \mathrm{~mL}$ de disolución de algas del género Scenedesmus. Las condiciones de luz y temperatura fueron las mismas que para el cultivo. Para cuantificar la biomasa inicial, al inicio de cada ensayo (T0) se tomaron al azar neonatos que fueron almacenados en formaldehido al $4 \%$ (Dumont et al. 1975). Una vez iniciados los ensayos y cada 5 días (T5, T10, T15 y T21), una de las cuatro repeticiones se retiró por cada tratamiento ensayado y los individuos fueron almacenados al igual que T0. La biomasa individual se cuantificó a través de la medida de la longitud de cada individuo. Los individuos $(n=\sim 400)$ fueron fotografiados, y su longitud $(\mu \mathrm{m})$ se midió con un microscopio óptico Leica DM500 con cámara digital Leica ICC50 HD para los tiempos T0, T5 y T10, y un microscopio estereoscópico Leica EZ4 para los tiempos T15 y T21. La longitud se tomó desde el borde del ojo hasta la base de la espina caudal de acuerdo a Giraudo et al. (2019) y en base a este parámetro se calculó la biomasa (peso seco en $\mu \mathrm{g}$ ) según lo indicado por Dumont et al. (1975) mediante la siguiente fórmula adaptada:

$$
\mathrm{B}=1.89 \mathrm{E}^{-6} \times\left(\mathrm{L}^{2.25}\right) \quad \text { Ecuación } 1
$$

donde B: biomasa individual $(\mu \mathrm{g})$ y L: largo del individuo $(\mu \mathrm{m})$.

Además se calculó la tasa de crecimiento somático diaria para cada tratamiento de acuerdo a Martin-Creuzburg y von Elert (2009), según la fórmula adaptada a continuación:

$$
\mathrm{C}=\left(\ln \mathrm{B}_{21}-\ln \mathrm{B}_{0}\right) / \mathrm{t} \quad \text { Ecuación } 2
$$

donde $\mathrm{C}$ : tasa de crecimiento diaria ( $\mu \mathrm{g} /$ día), $\mathrm{B}_{21}$ : biomasa individual a T21 $(\mu \mathrm{g}), \mathrm{B}_{0}$ : biomasa individual inicial $(\mu \mathrm{g})$ y t: tiempo de ensayo (21 días).

Las diferencias en los valores de biomasa entre tratamientos se evaluaron mediante (ANOVA) de un factor con contrastes a posteriori (Fisher) y mediante la prueba no paramétrica de Kruskal-Wallis en los casos donde no se cumplieron los supuestos. Todos los análisis se realizaron considerando un $P<0.05$ como criterio de significancia, utilizando el software Infostat. 


\section{Resultados}

\section{Calidad ambiental del agua}

La temperatura y la conductividad aumentaron gradualmente desde el ABEa hasta la BE (2.6-8.3 ${ }^{\circ} \mathrm{C}$ y $54-23342 \mu \mathrm{S} / \mathrm{cm}$, respectivamente), mientras que el oxígeno disuelto disminuyó levemente (15.87-11.36 $\mathrm{mg} / \mathrm{L}$ ) (Tabla 1). El pH se mantuvo cercano a la neutralidad en todos los casos y varió entre 6.6 y 7.9. Se registraron aumentos significativos en la concentración de $\mathrm{N}-\mathrm{NO}_{3}^{-}(P=0.019)$,

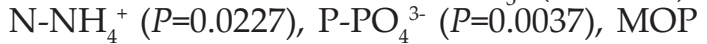
$(P=0.0003)$ y STS $(P=0.018)$ desde ABEa hacia el sitio BE. Las concentraciones de bacterias CT y CF aumentaron desde ABEa hasta ABEd y disminuyeron en el sitio BE.

\section{Bioensayos de toxicidad en Daphnia magna}

Toxicidad aguda. La sensibilidad del cultivo de Daphnia magna utilizando como tóxico de referencia al dicromato de potasio $\left(\mathrm{Cr}^{6+}\right)$ reveló un valor medio estimado de la $\mathrm{CL}_{50}$ de $279 \pm 40$ $\mu \mathrm{g} / \mathrm{L}$ a las 24 horas (intervalo de confianza $95 \%=220-245, R=0.99)$ y de $132 \pm 19 \mu \mathrm{g} / \mathrm{L}$ a las 48 horas (intervalo de confianza $95 \%=116$ $149, R=0.97)$. En los ensayos realizados con D. magna expuesta a las muestras de agua del $\mathrm{ABE}$ y la BE, la supervivencia a las 48 horas fue de $99.45 \%$, ya que sólo se registró en total 3 individuos muertos a las 24 horas en ABEd y BE. Por este motivo no fue posible estimar la $\mathrm{CL}_{50}$.
Toxicidad crónica. A lo largo de los 21 días de ensayo no hubo mortalidad de individuos de D. magna en los controles ni en los tres sitios del ABE. En uno de los dos bioensayos realizados con agua del sitio BE se produjo una mortalidad del $100 \%$ de los individuos entre los días 5 y 10 del tratamiento debido a la alta salinidad del agua recolectada.

La biomasa de los individuos de D. magna expuestos al agua de los diferentes tramos del $\mathrm{ABE}$ y $\mathrm{BE}$ a los cinco días de iniciado el ensayo (T5), no fue significativamente diferente entre tratamientos $(P=0.75)$ (Figura 2$)$. El valor medio de biomasa a T5 fue de 19.64 55.36 $\mu g(n=91)$. A los 10 días (T10), la biomasa fue significativamente diferente entre los tratamientos $(P=0.0003)$ : se observó un mayor crecimiento en los individuos expuestos al agua de ABEd $(48.03 \pm 10.97 \mu g ; n=17)$ y BE $(50.89 \pm 10.77 \mu g ; n=9)$, en comparación con ABEa (35.93 $\pm 11.82 \mu \mathrm{g} ; n=17)$ y el control (36.96 \pm $8.62 \mu \mathrm{g} ; n=18)$. Sin embargo, a partir de los 15 días (T15) se registró una tendencia diferente. Los mayores valores se presentaron en los individuos expuestos al tratamiento control (86.47 $\pm 11.15 \mu \mathrm{g} ; n=19 ; P=0.0008)$, mientras que los menores valores se observaron en los individuos del tratamiento BE (63.14 47.31 $\mu \mathrm{g} ; n=9)$. Al finalizar el ensayo a los 21 días (T21), la biomasa de los tratamientos control (93.28 $\pm 19.48 \mu \mathrm{g} ; n=18)$, ABEd (82.18 \pm 4.59 $\mu g ; n=19)$ y BE $(90.13 \pm 11.70 \mu g ; n=9)$ fue significativamente mayor $(P<0.0001)$ que la

Tabla 1. Valores medios \pm desvío estándar de los parámetros fisicoquímicos y microbiológicos determinados en las

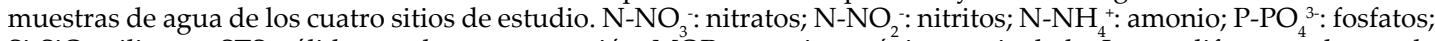
$\mathrm{Si}_{-} \mathrm{SiO}_{2}$ : silicatos; STS: sólidos totales en suspensión; MOP: materia orgánica particulada. Letras diferentes dentro de cada fila indican diferencias significativas $(P<0.05)$ entre sitios de estudio.

Table 1. Mean values \pm standard deviation of physicochemical and microbiological parameters determined in water

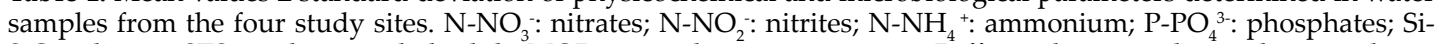
$\mathrm{SiO}_{2}$ : silicates; STS: total suspended solids; MOP: particulate organic matter. Different letters within each row indicate significant differences $(P<0.05)$ between study sites.

\begin{tabular}{|c|c|c|c|c|}
\hline Parámetro & $\mathrm{ABEa}$ & $\mathrm{ABEm}$ & ABEd & $\mathrm{BE}$ \\
\hline Temperatura $\left({ }^{\circ} \mathrm{C}\right)$ & 2.59 & 5.80 & 6.69 & 8.30 \\
\hline $\mathrm{pH}$ & $7.52 \pm 0.45$ & $7.16 \pm 0.22$ & $7.17 \pm 0.45$ & $7.25 \pm 0.92$ \\
\hline Conductividad $(\mu \mathrm{S} / \mathrm{cm})$ & $54 \pm 7.07$ & $105 \pm 43.84$ & $149 \pm 66.46$ & $23342 \pm 32326$ \\
\hline $\mathrm{O}_{2}$ disuelto $(\mathrm{mg} / \mathrm{L})$ & 15.87 & 13.41 & 12.16 & 11.36 \\
\hline $\mathrm{N}-\mathrm{NO}_{3}^{-}(\mathrm{mg} / \mathrm{L})$ & $0.013 \pm 0.001$ (a) & $0.053 \pm 0.024(\mathrm{ab})$ & $0.112 \pm 0.0924(b)$ & $0.084 \pm 0.022(b)$ \\
\hline $\mathrm{N}-\mathrm{NO}_{2}^{-}(\mathrm{mg} / \mathrm{L})$ & $0.013 \pm 0.002$ & $0.015 \pm 0.002$ & $0.013 \pm 0.003$ & $0.017 \pm 0.002$ \\
\hline $\mathrm{N}^{-\mathrm{NH}_{4}}{ }^{+}(\mathrm{mg} / \mathrm{L})$ & $0.001 \pm 0.002(\mathrm{a})$ & $0.064 \pm 0.033(\mathrm{ab})$ & $0.247 \pm 0.002(b)$ & $0.288 \pm 0.102(b)$ \\
\hline $\mathrm{P}^{-\mathrm{PO}_{4}^{3-}}(\mathrm{mg} / \mathrm{L})$ & $0.006 \pm 0.002(\mathrm{a})$ & $0.008 \pm 0.002(\mathrm{a})$ & $0.013 \pm 0.002(b)$ & $0.011 \pm 0.003(b)$ \\
\hline $\mathrm{Si}^{-\mathrm{SiO}_{2}}(\mathrm{mg} / \mathrm{L})$ & $0.471 \pm 0.060$ & $0.516 \pm 0.225$ & $0.614 \pm 0.030$ & $0.467 \pm 0.078$ \\
\hline STS (mg/L) & $600 \pm 0(a)$ & $9500 \pm 2969.8(b)$ & $13000 \pm 282.8(b)$ & $15000 \pm 4242.6(b)$ \\
\hline MOP (mg C/L) & $0.023 \pm 0.022(\mathrm{a})$ & $0.354 \pm 0.143(b)$ & $0.612 \pm 0.144(b)$ & $0.986 \pm 0.208(\mathrm{c})$ \\
\hline Bacterias coliformes totales (NMP/100 mL) & $<1.1$ & 94 & 1300 & $<18$ \\
\hline Bacterias coliformes fecales (NMP/100 mL) & $<1.1$ & 6.8 & 330 & $<18$ \\
\hline
\end{tabular}




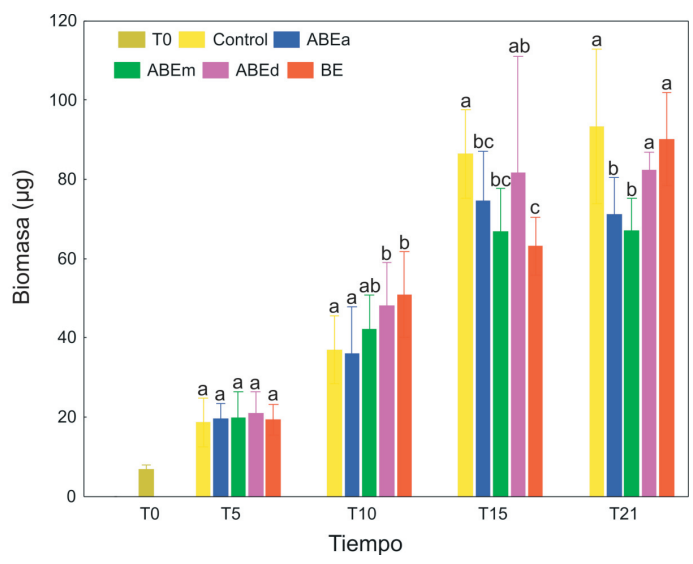

Figura 2. Valores medios \pm desvío estándar de la biomasa individual $(\mu \mathrm{g})$ como indicador de crecimiento a lo largo de 21 días en Daphnia magna expuesta al agua de distintos tramos del Arroyo Buena Esperanza (ABE) y de la Bahía Encerrada (BE). ABEa, ABEm y ABEd: ABE alto, medio y desembocadura, respectivamente. T0, T5, T10, T15 y T21 indican 0, 5, 10, 15 y 21 días de exposición, respectivamente. Letras distintas dentro de cada tiempo de exposición indican diferencias significativas $(P<0.05)$ entre tratamientos.

Figure 2. Mean values \pm standard deviation of individual biomass $(\mu \mathrm{g})$ as an indicator of growth in Daphnia magna during 21 days exposed to water from different sections of the Arroyo Buena Esperanza (ABE) and Bahía Encerrada (BE). ABEa, ABEm and ABEd: upper section, middle section and mouth, respectively. T0, T5, T10, T15 and T21 indicate $0,5,10,15$ and 21 days of exposure, respectively.

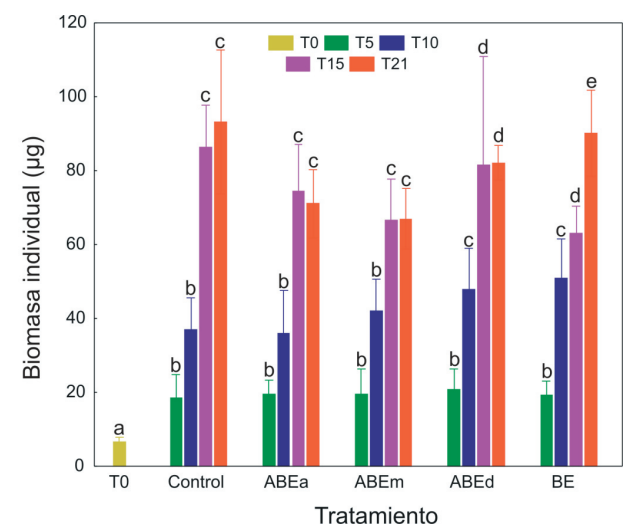

Figura 3. Valores medios \pm desvío estándar de la biomasa individual $(\mu \mathrm{g})$ como indicador de crecimiento dentro de cada tratamiento a lo largo de 21 días en Daphnia magna. ABE: Arroyo Buena Esperanza; BE: Bahía Encerrada; ABEa, ABEm y ABEd: ABE alto, medio y desembocadura, respectivamente. T0, T5, T10, T15 y T21 indican 0, 5, 10, 15 y 21 días de exposición, respectivamente. Letras distintas dentro de cada tratamiento indican diferencias significativas $(P<0.05)$ entre tiempos de exposición.

Figure 3. Mean values ‘ \pm standard deviation of individual biomass $(\mu \mathrm{g})$ as an indicator of growth in Daphnia magna for each treatment during 21 days of exposure. ABE: Arroyo Buena Esperanza; BE: Bahía Encerrada; ABEa ABEm and ABEd: upper section, middle section and mouth, respectively. T0, T5, T10, T15 and T21 indicate 0 , $5,10,15$ and 21 days of exposure, respectively. Different letters within each treatment indicate significant differences $(P<0.05)$ between exposure times. registrada en ABEa $(71.14 \pm 9.25 \mu \mathrm{g} ; n=18) \mathrm{y}$ ABEm (66.98 $\pm 8.21 \mu g ; n=19)$.

Al comparar los distintos tiempos de exposición dentro de cada tratamiento se observa que hay un aumento progresivo y significativo de la biomasa individual en todos los tratamientos $(P<0.05)$ (Figura $3)$. Las mayores diferencias se observan principalmente entre el T0, y T5 y T10, y entre T5 y T10 con T15 y T21. El crecimiento entre T15 y T21 fue solo significativo en el sitio BE, mientras que en el control y en los sitios del ABE el crecimiento fue menor y no significativo.

Las tasas de crecimiento somático obtenidas para los distintos tratamientos se observan en la Figura 4. La mayor tasa de crecimiento se registró en el control $(0.123 \mu \mathrm{g} /$ día $)$, seguida de las tasas obtenidas en los sitios ABEd (0.118 $\mu \mathrm{g} /$ día) y BE $(0.119 \mu \mathrm{g} /$ día $)$. Las menores tasas se presentaron en los tramos altos del ABE (ABEa: $0.111 \mu \mathrm{g} /$ día y ABEm: $0.106 \mu \mathrm{g} /$ día).

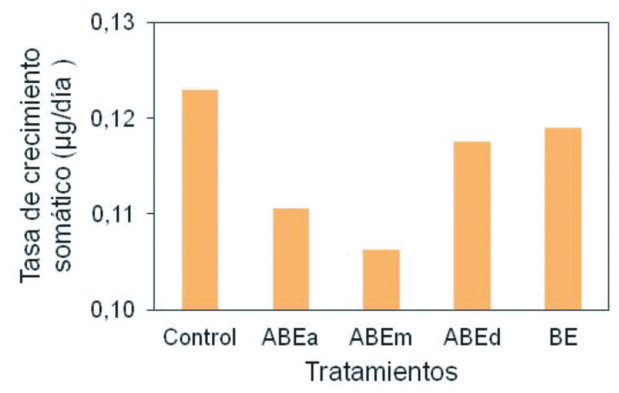

Figura 4. Tasa de crecimiento somático ( $\mu \mathrm{g} / \mathrm{día})$ en Daphnia magna luego de 21 días de exposición a los distintos tratamientos. ABE: Arroyo Buena Esperanza; BE: Bahía Encerrada; ABEa, ABEm y ABEd: ABE alto, medio y desembocadura, respectivamente.

Figure 4. Somatic growth rate ( $\mu \mathrm{g} /$ day) in Daphnia magna after 21 days of exposure to the different treatments. ABE: Arroyo Buena Esperanza; BE: Encerrada Bay; ABEa, ABEm and ABEd: upper section, middle section and mouth, respectively.

\section{Discusión}

El uso de los cursos de agua ha ido cambiando a través del tiempo con el desarrollo de la urbanización. De ser una fuente de provisión de agua y de actividades de recreación, los cursos de agua se convirtieron en vías de descarga de aguas de escurrimiento y efluentes hasta ser los receptores finales de una variedad de contaminantes (Rörig et al. 2007; Becouze-Lareure et al. 2016). El Arroyo Buena Esperanza (ABE) ha sido sometido a estos impactos durante más de dos décadas y se ha detectado que la calidad del agua y de sus sedimentos fluviales disminuye a medida 
que el curso de agua atraviesa el ejido urbano y desemboca en la costa de la Bahía Encerrada (Amin et al. 2011; Diodato et al. 2018, 2020). En el presente trabajo, los parámetros fisicoquímicos evaluados presentaron valores similares en cuanto al gradiente de calidad del $A B E$ registrado en trabajos previos, evidenciando que a medida que atraviesa la ciudad, el ABE experimenta un proceso de eutrofización cada vez más marcado hacia su desembocadura en la Bahía Encerrada. Los mayores valores de nitratos, amonio, fosfatos, materia orgánica particulada, sólidos totales en suspensión y bacterias coliformes fecales fueron encontrados principalmente en el tramo más bajo del arroyo (sitio ABEd), lo cual refleja un mayor impacto de la urbanización en este sitio. Al comparar los valores obtenidos con los registrados varios años atrás (Dirección General de Recursos Hídricos 2013; 2015; Diodato et al. 2018), la calidad mejoró notablemente en los tramos medio y bajo de la cuenca. Un ejemplo de ello se da en las concentraciones de amonio, fosfatos y materia orgánica particulada, que se redujeron en promedio 96, 97 y 86\%, respectivamente. Además, la concentración de bacterias coliformes disminuyó hasta tres órdenes de magnitud respecto de las halladas entre 2009 y 2015 (Dirección General de Recursos Hídricos 2013, 2015; Diodato et al. 2018). Estas disminuciones indican que hubo una reducción en los vertidos cloacales crudos al arroyo en el tramo bajo; se puede ver una mejor calidad de las aguas producto de las recientes obras de infraestructura llevadas adelante por la Dirección Provincial de Obras y Servicios Sanitarios de la provincia de Tierra del Fuego durante los dos últimos años.

Históricamente, la Bahía Encerrada ha presentado un alto impacto por la urbanización al estar muy influenciada por la desembocadura del ABE (Torres et al. 2009; Amin et al. 2011; Diodato et al. 2012). En el presente trabajo, el sitio BE se caracterizó por presentar, en algunos momentos del estudio, altos niveles de conductividad, mayores a los registrados durante el período 20052015 (Torres et al. 2009; Diodato et al. 2018) y asociados a un mayor intercambio hídrico con la Bahía Ushuaia. Este aumento en el recambio de agua es producto del reciente reacondicionamiento de la conexión entre ambos cuerpos de agua (Abascal 2016). Este mayor intercambio, cuya magnitud varía de acuerdo al estado de la marea, explica las diferencias de conductividad y salinidad encontradas durante el estudio, a pesar de haber recolectado las muestras de agua en bajamar. Estas diferencias se vieron reflejadas en los resultados de los ensayos crónicos, ya que como se mencionó previamente, uno de los ensayos presentó una mortalidad del 100\% luego del quinto día de exposición debido a la alta salinidad.

Por otro lado, el detrimento en la calidad del agua del ABE y la BE ha generado impactos negativos sobre la biota. Biancalana y Torres (2011) reportaron una alta abundancia en la BE del copépodo Eurytemora americana, una especie oportunista y bioindicadora de ambientes con alta carga orgánica. De acuerdo con Zagarola et al. (2017) la biodiversidad de macroinvertebrados bentónicos y el oxígeno disuelto disminuyeron en el tramo bajo del arroyo respecto a la zona alta de la cuenca del ABE, mientras que Granitto et al. (2021) encontraron una correlación positiva entre el aumento de nutrientes y la clorofila perifítica en los tramos bajos del ABE, evidenciando un proceso de eutrofización.

El uso del cladócero Daphnia magna en bioensayos de toxicidad como herramienta para evaluar la calidad del agua del ABE mostró resultados satisfactorios. La respuesta general de la especie ensayada fue adecuada y confiable, ya que los resultados obtenidos en los ensayos de sensibilidad al $\mathrm{K}_{2} \mathrm{Cr}_{2} \mathrm{O}_{7}$ como tóxico de referencia fueron acordes a los previstos. La $\mathrm{CL}_{50}$ a 24 horas estimada $(279 \mu \mathrm{g} \mathrm{Cr} / \mathrm{L})$, que corresponde a una concentración de 1.58 mg $\mathrm{K}_{2} \mathrm{Cr}_{2} \mathrm{O}_{7} / \mathrm{L}$, fue similar al valor encontrado (1.28 $\left.\mathrm{mg} \mathrm{K}_{2} \mathrm{Cr}_{2} \mathrm{O}_{7} / \mathrm{L}\right)$ por Alves y Silvano (2006) para la misma especie. Para las 48 horas se obtuvo una $\mathrm{CL}_{50}$ de $0.75 \mathrm{mg} \mathrm{K}_{2} \mathrm{Cr}_{2} \mathrm{O}_{7} / \mathrm{L}$ (132 $\mu \mathrm{g} \mathrm{Cr} / \mathrm{L})$, levemente superior al valor de 0.64 $\mathrm{mg} \mathrm{K} \mathrm{Cr}_{2} \mathrm{O}_{7} / \mathrm{L}$ provisto por Gopi et al. (2012) y de $0.47 \mathrm{mg} \mathrm{K}_{2} \mathrm{Cr}_{2} \mathrm{O}_{7} / \mathrm{L}$ señalado por Verma (2008). La similitud entre las $\mathrm{CL}_{50}$ calculadas y las obtenidas en otros estudios asegura que el cultivo desarrollado en laboratorio fue óptimo para llevar a cabo los bioensayos de toxicidad con las aguas del ABE y BE.

En el presente estudio, la exposición aguda al agua del ABE y de la BE no afectó la supervivencia de los individuos de D. magna durante las 48 horas de exposición. Este resultado indica que las muestras recolectadas no fueron tóxicas para D. magna, lo cual concuerda con lo hallado en otros estudios. Por ejemplo, Baun et al. (2003), Christensen et al. (2006), Becouze-Lareure et al. (2016) y Giraudo et al. (2019) analizaron muestras completas de 
agua de cursos urbanos contaminados con efluentes domiciliarios sobre neonatos de la misma especie, y tampoco hallaron toxicidad sobre los organismos.

Ante la ausencia de letalidad del agua del ABE y la BE, una forma de evaluar la posible toxicidad a largo plazo fue analizar el crecimiento (en forma de cambio en biomasa individual) como parámetro de evaluación crónica (Ferrari 2006; Giraudo et al. 2019). El crecimiento de los individuos expuestos a lo largo de los 21 días de ensayo varió de acuerdo al sitio de exposición y al tiempo ensayado. Al inicio (T5), el crecimiento fue parejo en todos los tratamientos, pero a partir de los 10 días de ensayo las diferencias se hicieron más marcadas. Como las condiciones generales de cultivo fueron las mismas, la diferencia se debe a la calidad del agua a la cual fueron expuestos. A los 10 días de ensayo, los individuos expuestos al agua de los sitios más impactados por la urbanización (ABEd y BE) presentaron mayor crecimiento en comparación con el sitio sin impacto (ABEa) y el control. Los sitios más impactados presentaron mayores niveles de materia orgánica, de sólidos suspendidos y de nutrientes de $\mathrm{N}$ y $\mathrm{P}$. Esta mayor disponibilidad de sólidos en suspensión y de nutrientes disueltos podría haber favorecido el crecimiento de D. magna mediante la estimulación en la alimentación, debido a que la especie es filtradora. Aunque la alimentación provista en los distintos tratamientos fue similar en composición y concentración, la mayor disponibilidad de nutrientes propia de las muestras ensayadas podría haber favorecido un mayor crecimiento del fitoplancton, propiciando así una mayor tasa de filtración e ingestión y estimulando el aumento en biomasa (Sterner et al. 1993). Sin embargo, Serra et al. (2019) afirman que la exposición crónica (7 días) a amonio, nitritos y, en menor medida, a fosfatos, puede generar disminuciones en las tasas de filtración de D. magna, lo que afecta así su crecimiento somático; esto contradice nuestra propuesta. Sin embargo, las concentraciones a las que observaron estos efectos son de mayor magnitud que las concentraciones de nutrientes encontradas en el ABE, por lo cual la respuesta podría depender de las concentraciones de nutrientes presentes en las muestras.

El aumento en la biomasa en los individuos expuestos a los sitios más impactados concuerda con lo reportado por Giraudo et al. (2019), quienes observaron mayor crecimiento de los neonatos de D. magna después de 13 días de exposición a aguas urbanas no tratadas, y lo atribuyeron a las mayores concentraciones de $\mathrm{Cr}$ y Zn, así como a otros potenciales contaminantes presentes en las aguas urbanas ensayadas. Por otro lado, Steinkey et al. (2018) encontraron que distintas concentraciones del medicamento gemfibrozil en agua favorecía el crecimiento de D. magna en 21 días de tratamiento cuando la cantidad de alimento era suficientemente alta. Como resulta de lo expuesto anteriormente, tanto la mortalidad como las variaciones en las tasas de crecimiento pueden verse afectadas por muchos otros compuestos químicos que no pudieron evaluarse en este estudio. Debido a que las aguas residuales urbanas son una mezcla compleja de compuestos tales como metales pesados y productos de cuidado personal de preocupación emergente, los efectos sobre el crecimiento de $D$. magna no pudieron ser relacionados directamente con una clase particular de contaminantes. La estimulación del crecimiento hallada en los organismos expuestos a ABEd y BE podría deberse no sólo al aporte de nutrientes sino también a la presencia de otros compuestos que podrían influir en la respuesta (Ebele et al. 2017; Steinkey et al. 2018; Giraudo et al. 2019).

Otro parámetro de evaluación crónica reportado por Kosmala et al. (1999) y Tamura et al. (2017) es la reproducción. Estos autores encontraron que la reproducción en Ceriodaphnia dubia aumentó frente a la exposición durante 7-8 días al agua de arroyos urbanos impactados por descargas cloacales. El incremento en nutrientes por la actividad bacteriana en las aguas contaminadas es la causa del aumento en el número de neonatos (Kosmala et al. 1999; Tamura et al. 2017), lo cual concuerda con lo propuesto en el presente trabajo.

Luego de los primeros 10 días de ensayo (registro a los 15 y a los 21 días), los individuos del tratamiento control crecieron de forma equivalente a los ensayados con agua de ABEd y BE, mientras que los individuos expuestos al agua del ABEa y ABEm permanecieron con una menor biomasa final. Las tasas de crecimiento reflejaron este mismo patrón, donde los sitios con menor impacto (ABEa y ABEm) presentaron las menores tasas y las correspondientes a los sitios impactados fueron más similares a las tasas del control. El medio de cultivo estandarizado para D. magna utilizado en el tratamiento control es el ideal 
para el óptimo crecimiento de los individuos; les aporta los nutrientes necesarios para un mejor desarrollo (USEPA 2002). A pesar de que el agua de ABEd y BE contenía mayor concentración de nutrientes después de los 10 días de ensayo, el aumento en biomasa de D. magna se asemejó al del control. Tal como se mencionó previamente, en esta instancia de cultivo la presencia y permanencia en el tiempo de otras sustancias en el agua no determinadas en este estudio (tales como metales o productos farmacéuticos) podrían afectar también el grado de aumento en la biomasa.

Recomendamos tener en cuenta los días totales de ensayo en la evaluación de la biomasa como indicador de efectos subletales ya que entre los días 15 y 21 observamos un mantenimiento en el crecimiento en los sitios impactados probablemente a causa del inicio de la reproducción partenogenética (datos no presentados). Consideramos que finalizar los ensayos crónicos a los 15 días es el momento óptimo para determinar cambios en el crecimiento de D. magna utilizando la biomasa individual como indicador.

En base a los resultados obtenidos, no detectamos toxicidad aguda ni crónica sobre el cladócero D. magna frente a la exposición al agua de un arroyo urbano. De todos modos, los cambios en biomasa respecto a sitios no impactados y al control resultaron ser una herramienta de diagnóstico ambiental aplicable de forma comparativa entre puntos de un mismo arroyo y de manera complementaria al monitoreo ambiental.

Agradecimientos. Los autores agradecen al Centro de Investigaciones del Medio Ambiente de la Universidad Nacional de La Plata por la provisión de los primeros organismos de cultivo de Daphnia magna y a la Dra. P. Rodríguez por sus valiosos comentarios sobre el manuscrito. Este trabajo fue financiado por el proyecto PIDUNTDF B 04/2017 otorgado por la Universidad Nacional de Tierra del Fuego y formó parte del Trabajo Final de Tesis de Grado de Antonela Albizzi.

\section{REFERENCIAS}

Abascal, L. 2016. Estudio de factibilidad para el saneamiento y la recuperación ambiental del frente marítimo y espacio acuático de la Bahía Encerrada. Informe Final Consolidado. Municipalidad de Ushuaia. Tierra del Fuego, Argentina.

Alves, A. C. B., and J. Silvano. 2006. Avaliação da sensibilidade de Daphnia magna Straus, 1820 (Cladócera, Crustácea) ao dicromato de potássio. Revista do Instituto Adolfo Lutz (Impresso) 65(1):59-61.

Amin, O., L. Comoglio, C. Spetter, C. Duarte, R. Asteasuain, R. H. Freije, and J. Marcovecchio. 2011. Assessment of land influence on a high-latitude marine coastal system: Tierra del Fuego, southernmost Argentina. Environmental Monitoring and Assessment 175(1-4):63-73. https://doi.org/10.1007/s10661-010-1493-5.

Angerville, R., Y. Perrodin, C. Bazin, and E. Emmanuel. 2013. Evaluation of ecotoxicological risks related to the discharge of combined sewer overflows (CSOs) in a periurban river. International Journal of Environmental Research and Public Health 10:2670-2687. https://doi.org/10.3390/ijerph10072670.

APHA- AWWA- WEF. 2017. Standard Methods for the Examination of Water and Wastewater. Washington.

Baun, A., A. M. Christensen, and F. Nakajima. 2003. Ecotoxic effects of wet weather discharges in an urban stream. Diffuse Pollution Conference Dublin.

Becouze-Lareure, C., L. Thiebaud, C. Bazin, P. Namour, P. Breil, and Y. Perrodin. 2016. Dynamics of toxicity within different compartments of a peri-urban river subject to combined sewer overflow discharges. Science of the Total Environment 539:503-514. https://doi.org/10.1016/j.scitotenv.2015.08.128.

Biancalana, F., and A. I. Torres. 2011. Variations of mesozooplankton composition in a eutrophicated semi-enclosed system (Encerrada Bay, Tierra del Fuego, Argentina). Brazilian Journal of Oceanography 59(2):195-199. https://doi.org/ 10.1590/S1679-87592011000200008.

Castillo Morales, G. 2004. Ensayos toxicológicos y métodos de evaluación de calidad de aguas. Estandarización, intercalibración, resultados y aplicaciones. First edition. IMTA, México.

Castro-Mejía, J., J. A. Ocampo-Cervantes, G. Castro-Mejía, I. Cruz-Cruz, M. C. Monroy-Dosta, and D. Becerril-Cortes. 2016. Laboratory production of Daphnia magna (Straus 1820) fed with microalgae and active dry yeast. Journal of Entomology and Zoology Studies 4(2):548-553.

Chalmers, A. T., P. C. Van Metre, and E. Callender. 2007. The chemical response of particle-associated contaminants in aquatic sediments to urbanization in New England, U.S.A. Journal of Contaminant Hydrology 9:4-25. https: //doi.org/10.1016/j.jconhyd.2006.08.007.

Chantanachat, S., and H. C. Bold. 1962. Phycological Studies II. Some algae from arid soils. University of Texas Publication $\mathrm{N}^{\circ}$ 6218. Austin, Texas.

Christensen, A. M., F. Nakajima, and A. Baun. 2006. Toxicity of water and sediment in a small urban river (Store Vejleå, Denmark). Environmental Pollution 144(2):621-625. https://doi.org/10.1016/j.envpol.2006.01.032.

Damásio, J., R. Tauler, E. Teixidó, M. Rieradevall, N. Prat, M. C. Riva, A. M. N. M. Soares, and C. Barata. 2008. Combined use of Daphnia magna in situ bioassays, biomarkers and biological indices to diagnose and identify environmental 
pressures on invertebrate communities in two Mediterranean urbanized and industrialized rivers (NE Spain). Aquatic Toxicology 87(4):310-320. https://doi.org/10.1016/j.aquatox.2008.02.016.

Díaz Báez, M., Y. Pica Granados, and A. Ronco. 2004. Ensayo de toxicidad aguda con el cladócero Daphnia magna. Pp. 52 63 in G. Castillo Morales (ed.). Ensayos toxicológicos y métodos de evaluación de calidad de aguas. IMTA, México.

Diodato S., L. Comoglio, C. Camilión, and O. Amin. 2012. Responses of the resident rocky crab (Halicarcinus planatus, Decapoda) to natural stressors and effluent discharges in Ushuaia Bay, Tierra del Fuego, Argentina. Journal of Experimental Marine Biology and Ecology 436-437:11-18. https://doi.org/10.1016/j.jembe.2012.08.011.

Diodato, S. 2013. Respuestas de especies locales a procesos de eutrofización en la zona costera próxima a la ciudad de Ushuaia, Tierra del Fuego. Tesis Doctoral. Universidad Nacional del Sur, Bahía Blanca. Argentina. Pp. 221.

Diodato, S., L. Comoglio, A. Moretto, and J. Marcovecchio. 2018. Dinámica e impacto de la eutrofización por aportes urbanos en las cuencas hídricas y zona costera de la ciudad de Ushuaia, Tierra del Fuego. Pp. 468-475 in Abraham, E., R. Quintana and G. Mataloni (eds.). Aguas + Humedales, Serie Futuros. UNSAM EDITA, Buenos Aires.

Diodato, S., G. González Garraza, R. Mansilla, A. Moretto, J. Escobar, M. Méndez-López, A. Gómez-Armesto, J. Marcovecchio, and J. Nóvoa-Muñoz. 2020. Quality changes of fluvial sediments impacted by urban effluents in Ushuaia, Tierra del Fuego, southernmost Patagonia. Environmental Earth Sciences 79:481. https://doi.org/10.1007/ $\underline{\text { s12665-020-09236-4. }}$.

Dirección General De Recursos Hídricos. 2013. Informe de calidad de aguas de la ciudad de Ushuaia, octubre de 2013. Informe Técnico. Secretaría de Ambiente, Desarrollo Sostenible y Cambio Climático. Tierra del Fuego, Argentina.

Dirección General De Recursos Hídricos. 2015. Informe de monitoreo de calidad de agua - Plan Hidroambiental de la ciudad de Ushuaia sector este. Octubre 2015. Secretaría de Ambiente, Desarrollo Sostenible y Cambio Climático. Tierra del Fuego, Argentina.

Dumont, H. J., I. Van De Velde, and S. Dumont. 1975. The dry weight estimate of biomass in a selection of Cladocera, Copepoda and Rotifera from the plankton, periphyton and benthos of continental waters. Oecologia 19(1):75-97. https://doi.org/10.1007/BF00377592.

Ebele A. J., M. A. E. Abdallah, and S. Harrad. 2017. Pharmaceuticals and personal care products (PPCPs) in the freshwater aquatic environment. Emerging Contaminants 3:1-16. https://doi.org/10.1016/j.emcon.2016.12.004.

Ferrari, L. 2006. Generalidades sobre ecotoxicología. Pp. 393-421 in Carballo M. and M. Mudry (eds.). Genética Toxicológica. Editorial Los Cuatro Vientos, Buenos Aires, Argentina.

Giraudo, M., M. Douville, and M. Houde. 2015. Chronic toxicity evaluation of the flame retardant tris (2-butoxyethyl) phosphate (TBOEP) using Daphnia magna transcriptomic response. Chemosphere 132:159-165. https://doi.org/10.1016/ j.chemosphere.2015.03.028.

Giraudo, M., T. L. Colson, M. Pilote, C. Gagnon, P. Gagnon, and M. Houde. 2019. A major release of urban untreated wastewaters in the St. Lawrence River (Quebec, Canada) altered growth, reproduction, and redox status in experimentally exposed Daphnia magna. Ecotoxicology 28(7):843-851. https://doi.org/10.1007/s10646-019-02084-4.

Göbel, P., C. Dierkes, and W. G. Coldewey. 2007. Storm water runoff concentration matrix for urban areas. Journal of Contaminant Hydrology 91:26-42. https://doi.org/10.1016/j.jconhyd.2006.08.008.

Gopi, R. A., S. Ayyappan, G. Chandrasehar, V. Krishna, and A. Goparaju. 2012. Effect of potassium dichromate on the survival and reproduction of Daphnia magna. Bulletin of Environment, Pharmacology and Life Sciences 1(7):89-94. https://doi.org/10.5402/2012/247072.

Granitto, M., S. Diodato, and P. Rodríguez. 2021. Water quality index including periphyton chlorophyll- $a$ in forested urban watersheds from Tierra del Fuego (Argentina). Ecological Indicators 126:107614. https://doi.org/10.1016/ j.ecolind.2021.107614

Iturraspe, R. 2007. Report on basin response for Argentina. Deliverable D18 EPIC ORCE. Tierra del Fuego, Argentina.

Kosmala, A., S. Charvet, M. C. Roger, and B. Faessel. 1999. Impact assessment of a wastewater treatment plant effluent using instream invertebrates and the Ceriodaphnia dubia chronic toxicity test. Water Research 33(1):266-278. https: //doi.org/10.1016/S0043-1354(98)00176-6.

Le Moal, M., C. Gascuel-Odoux, A. Ménesguen, Y. Souchon, C. Étrillard, A. Levain, F. Moatar, A. Pannard, P. Souchu, A. Lefebvre, and G. Pinay. 2019. Eutrophication: A new wine in an old bottle? Science of the Total Environment 651 : 1-11. https://doi.org/10.1016/j.scitotenv.2018.09.139.

Marcogliese, D. J., C. Blaise, D. Cyr, Y. De Lafontaine, M. Fournier, F. Gagné, C. Gagnon, and C. Hudon. 2015. Effects of a major municipal effluent on the St. Lawrence River: A case study. Ambio 44(4):257-274. https://doi.org/10.1007/ s13280-014-0577-9.

Martin-Creuzburg, D., and E. Von Elert. 2009. Good food versus bad food: the role of sterols and polyunsaturated fatty acids in determining growth and reproduction of Daphnia magna. Aquatic Ecology 43(4):943-950. https://doi.org/ 10.1007/s10452-009-9239-6.

Martínez-Jerónimo, F., J. Rodríguez-Estrada, and L. Martínez-Jerónimo. 2008. Daphnia exilis Herrick, 1895 (Crustacea: Cladocera): Una especie zooplanctónica potencialmente utilizable como organismo de prueba en bioensayos de toxicidad aguda en ambientes tropicales y subtropicales. Revista Internacional de Contaminación Ambiental 24(4): 153-159.

OECD. 2008. Guidelines for Testing of Chemicals. Daphnia magna Reproduction Test OECD 211. OECD Publishing, Paris.

Rörig, L., J. Tundisi, C. Schettini, J. Pereira-Filho, J. Menezes, T. Almeida, S. Urban, C. Radetski, R. Sperb, C. Stramosk, R. Macedo, M. Castro-Silva, and J. Pérez. 2007. From a water resource to a point pollution source: the daily journey of a 
coastal urban stream. Brazilian Journal of Biology 67(4):597-609. https://doi.org/10.1590/S1519-69842007000400003.

Scholes, L., A. Baun, M. Seidl, E. Eriksson, M. Revitt, and J. Mouchel. 2007. Assessment of storm water ecotoxicity using a battery of biotests. Pp. 399-410 in Morrison G. and S. Rauch (eds.). Highway and Urban Environment: Proceedings of the 8th Highway and Urban Environment Symposium. Springer. https://doi.org/10.1007/978-1-4020-6010-6_35.

Serra, T., M. Soler, N. Pous, and J. Colomer. 2019. Daphnia magna filtration, swimming and mortality under ammonium, nitrite, nitrate and phosphate. Science of the Total Environment 656:331-337. https://doi.org/10.1016/ j.scitotenv.2018.11.382.

Steinkey, D., E. Lari, S. Woodman, K. Luong, C. Wong, and G. Pyle. 2018. Effects of gemfibrozil on the growth, reproduction, and energy stores of Daphnia magna in the presence of varying food concentrations. Chemosphere 192: 75-80. https://doi.org/10.1016/j.chemosphere.2017.10.124.

Sterner, R. W., D. D. Hagemeier, W. L. Smith, and R. F. Smith. 1993. Phytoplankton nutrient limitation and food quality for Daphnia. Limnol. Oceanogr. 38(4):857-871. https://doi.org/10.4319/lo.1993.38.4.0857.

Strauss, H. E. 1820. Memoire sur les Daphnia de la clase des Crustaces (seconde partie). Memoires du Musee Royale d'Histoire Naturelle de Belgique 6:149-162.

Strickland, J., and T. Parsons. 1972. A Practical Handbook of Seawater Analysis. Fisheries Research Board of Canada 167.

Tamura, I., Y. Yasuda, K. I. Kagota, S. Yoneda, N. Nakada, V. Kumar, Y. Kameda, K. Kimura, N. Tatarazako, and H. Yamamoto. 2017. Contribution of pharmaceuticals and personal care products (PPCPs) to whole toxicity of water samples collected in effluent-dominated urban streams. Ecotoxicology and Environmental Safety 144:338-350. https: //doi.org/10.1016/j.ecoenv.2017.06.032.

Ten Berge, W. F. 1978. Breeding Daphnia magna. Hydrobiologia 59(2):121-123. https://doi.org/10.1007/BF00020772.

Torres, A., M. Gil, O. Amin, and J. Esteves. 2009. Environmental characterization of an eutrophicated semi-enclosed system: nutrient budget (Encerrada Bay, Tierra del Fuego Island, Patagonia, Argentina). Water, Air, and Soil Pollution 204:259-270. https://doi.org/10.1007/s11270-009-0042-8.

USEPA. 2002. Methods for Measuring the Acute Toxicity of Effluents and Receiving Waters to Freshwater and Marine Organisms. U.S. Environmental Protection Agency. Washington.

Urciuolo, A., and R. Iturraspe. 2005. Ordenamiento hídrico de las cuencas de fuentes aptas para provisión de agua potable a la ciudad de Ushuaia. Proccedings of the $20^{\text {th }}$ Congreso Nacional del Agua. May 9-14. 2005. Mendoza, Argentina.

Van Leeuwen, J., J. Awad, B. Myers, and D. Pezzaniti. 2019. Introduction to Urban Stormwater: A Global Perspective. Pp. 1- 28 in Jegatheesan V., A. Goonetilleke, J. van Leeuwen, J. Kandasamy, D. Warner, B. Myers, M. Bhuiyan, K. Spence and G. Parker (eds.). Urban Stormwater and Flood Management. Applied Environmental Science and Engineering for a Sustainable Future. Springer Nature Switzerland.

Verma, Y. 2008. Toxicity assessment of pulp-paper mill effluents employing Daphnia bioassay. Japanese Journal of Environmental Toxicology 11(2):151-156.

Zagarola, J., G. Martínez Pastur, M. Lopez, and C. Anderson. 2017. Assessing the effects of urbanization on streams in Tierra del Fuego. Ecología Austral 27:45-54. https://doi.org/10.25260/EA.17.27.1.0.417. 